\title{
Desenvolvimento de Ophyra albuquerquei Lopes (Diptera, Muscidae) em condições de laboratório ${ }^{1}$
}

\author{
Rodrigo Ferreira Krüger ${ }^{1}$ \\ Paulo Bretanha Ribeiro ${ }^{2}$ \\ Claudio José Barros de Carvalho ${ }^{3}$
}

\begin{abstract}
Development of Ophyra albuquerquei Lopes (Diptera, Muscidae) in laboratory conditions. Ophyra albuquerquei may often be a potential predator of Musca domestica Linnaeus, 1758 in poultry and pig houses in southern Brazil. Here we address the developmental period of immatures stages and their viability. To obtain eggs, larvae and pupae, a colony was established in the laboratory. Adult flies were fed on a diet comprising two parts dry milk, two parts sugar and one part fish flour. Larval diet comprised one part fish flour and one part sawdust and water. The eggs, larvae and pupae were incubated at $26^{\circ} \pm 1{ }^{\circ} \mathrm{C}, 75 \% \pm 10 \%$ R.H. and $12 \mathrm{~h}$ photoperiod. The egg to adult cycle took $573 \mathrm{~h}$ with a total viability of $64 \%$. The shortest of the stages was the egg stage, which lasted about $20 \mathrm{~h}$. The larval stage had the lowest viability (about $82 \%$ ) and longest time interval $(279 \mathrm{~h})$. Overall, the results of this study show that $O$. albuquerquei can be readily maintained in the laboratory.
\end{abstract}

KeYwords. Biological control; immature development; Muscidae; Ophyra albuquerquei.

\section{INTRODUÇÃO}

As espécies de Ophyra Robineau-Desvoidy, 1830 (Muscidae, Azeliinae), no estágio de larva de terceiro ínstar, são predadoras facultativas e geralmente estão associadas com fezes humanas (D’Almeida 1988, 1989), fezes de animais destinados à produção, como aves de corte e poedeiras ( AxTELL 1999), fezes de suínos (Avancini \& Silveira 2000), cadáveres humanos (CARVALHO et al. 2000) e de outros animais (BOHART \& GRESSIT 1951; TANTAWI et al. 1996). Nestes substratos as larvas das espécies de Ophyra freqüentemente estão envolvidas na predação de outras larvas, principalmente as de Musca domestica Linnaeus, 1758 e Stomoxys calcitrans Linnaeus, 1758, além de sarcofagídeos e califorídeos (ANDERson \& POORBAUGH 1964; PeCK 1969; S KIDMORe 1985; Geden et al. 1988).

Nos sistemas de produção de aves e suínos, Ophyra aenescens (Wiedemann,1830) e Ophyra ignava (Harris, 1780) são usadas no controle das populações de $M$. domestica na Alemanha (BetKe et al. 1989; SCHUMAnN 1989) e nos Estados
Unidos (Youngman et al. 1991). Da mesma forma, na África do Sul (Olckers \& Hulley 1984) e na Bulgária (Tsankova \& LuvchiEv 1993) foi sugerida a possibilidade de utilização também de larvas de Ophyra capensis (Wiedemann,1818) para o controle de M. domestica.

No Brasil, ocorrem cinco espécies de Ophyra: O. aenescens, O. albuquerquei Lopes, 1985, O. capensis, O. chalcogaster (Wiedemann, 1824) e O. solitaria Albuquerque, 1958 (PAmplona \& Couri 1989; Carvalho et al. 1993). Destas cinco espécies, duas ocorrem exclusivamente no Brasil, $O$. solitaria e $O$. albuquerquei. Pouco se conhece a respeito da biologia destas duas espécies, com exceção da flutuação populacional na região de Pelotas, Rio Grande do Sul, onde O. albuquerquei possui maior frequiência de ocorrência (RibeIRo et al. 2000b) quando comparada às outras espécies de Ophyra, estando mais associada ao ambiente silvestre (CostA et al. 2000).

O estudo da biologia de uma espécie é um campo amplo e diverso, onde os conhecimentos básicos são geralmente obtidos em laboratório sob condições uniformes de

1. Contribuição ${ }^{\circ} 1406$ do Departamento de Zoologia, Universidade Federal do Paraná.

2. Programa de Pós-Graduação em Ciências Biológicas/Entomologia, Departamento de Zoologia, Universidade Federal do Paraná. Caixa Postal 19020, 81531-980 Curitiba - PR. Bolsista da CAPES. Endereço Eletrônico: rfkruger@bio.ufpr.br

3. Departamento de Microbiologia e Parasitologia, Universidade Federal de Pelotas. Caixa Postal 354, 96010-900, Pelotas - RS. Endereço Eletrônico: bretanha@ufpel.tche.br

4. Departamento de Zoologia, Universidade Federal do Paraná. Caixa Postal 19020, 81531-980, Curitiba-PR. Bolsista do CNPq. Endereço Eletrônico: cjbcarva@ufpr.br 
temperatura, umidade relativa do ar e fotoperíodo. Isto permite o acompanhamento de todos os estágios do inseto em qualquer época do ano. Para facilitar o manejo, sem depender da utilização de substratos naturais que demandam oferta constante no ambiente, há necessidade da confecção de dietas artificiais. Neste caso, a dieta deve permitir: alta viabilidade dos estágios imaturos e produção de adultos com boa capacidade reprodutiva (PARRA 1996).

YounGMAN et al. (1991) alcançaram boa produção massal de O. aenescens utilizando uma dieta composta de uma parte de farinha de carne misturada com duas partes de leite em pó e duas partes de açúcar para os adultos, adicionando farinha de carne e osso ou farinha de peixe para as larvas. HogsetTE \& WASHINGTON (1995) somente obtiveram sucesso na criação de $O$. aenescens em dieta vegetal, quando adicionaram farinha de carne e osso, demonstrando a necessidade de altas taxas de proteínas na dieta desta espécie para a sua criação. Este fato foi levado em conta por RiBEIRo et al. (2000a) que criaram larvas de $O$. aenescens em uma dieta de farinha de peixe, farinha de trigo e serragem umedecida, em uma proporção de 50\%, $20 \%$ e $30 \%$, oferecendo aos adultos a mesma dieta de Youngman et al. (1991).

Além da dieta, a temperatura é outro fator que atua no desenvolvimento dos insetos, sendo que a faixa ótima de desenvolvimento de qualquer inseto está em torno de $15^{\circ}$ a $38^{\circ} \mathrm{C}$, sendo a temperatura de $25^{\circ} \mathrm{C}$, o ponto ótimo de desenvolvimento (SILVEIRA-NETO et al., 1976). Para O. aenescens a faixa de temperatura mais aconselhável ao seu desenvolvimento ocorre entre $20^{\circ}$ e $30^{\circ} \mathrm{C}$ (RIBEIRo et al. 2000a).

A implementação do controle biológico no manejo integrado de $M$. domestica em granjas avícolas e de suínos, deve acontecer utilizando espécies que naturalmente ocorram no mesmo ambiente desta praga, diminuindo desta forma, o perigo da introdução de espécies.

O objetivo deste trabalho foi estimar os períodos de desenvolvimento dos estágios imaturos, bem como a viabilidade destas fases em $O$. albuquerquei sob condições de laboratório em dieta artificial.

\section{MATERIAL EMÉTODOS}

Uma colônia de $O$. albuquerquei foi estabelecida no laboratório, em câmara climatizada, a partir de adultos capturados em uma granja avícola no campus da Universidade Federal de Pelotas (UFPel) na cidade de Capão do Leão, RS (31 $45^{\circ}$ ' $48^{\prime}$ ' $\mathrm{S}$ e $52^{\circ} 29^{\prime} 02^{\prime}$ 'W), para obtenção de ovos, larvas e pupas. Os adultos foram acondicionados em gaiolas com 30x30x30 cm e alimentados com leite em pó, açúcar refinado e farinha de peixe, em uma proporção de 2:2:1, oferecendo-se água em copos de Becker com espuma de poliestireno cobrindo a superfície do líquido. A ração e a água foram oferecidas ad libitum.

Para a obtenção de ovos foram colocadas, no interior das gaiolas, placas com meio de cultura composto de farinha de peixe e serradura, em uma proporção de 1:1, e água em quantidade suficiente para tornar o meio pastoso. Neste meio, as larvas desenvolveram-se até a fase de pós-alimentação, quando eram transferidas para serragem úmida na qual passariam esta fase e os estágios de pré-pupa e pupa. A renovação da colônia foi a partir de posturas da própria colônia.

Os ovos, larvas e pupas foram incubados em câmara de germinação à temperatura de $26^{\circ} \pm 1^{\circ} \mathrm{C}$, com umidade relativa de $75 \% \pm 10 \%$ e fotoperíodo de 12 horas. Os ovos, larvas e pupas utilizados na estimativa do período de desenvolvimento e viabilidade pertenciam a $\mathrm{F}_{4}$ (quarta geração). Para a estimativa do período de incubação e de sua viabilidade, foram utilizados 275 ovos, observados de hora em hora, 12 horas após ter sido efetuada a oviposição. Para cada ínstar do estágio larval foram utilizadas 200 larvas para estimar o período de desenvolvimento e a viabilidade. As larvas de primeiro e segundo ínstares foram observadas em intervalos de seis horas. As larvas de terceiro ínstar, na fase alimentar, foram observadas em intervalos de 12 horas. A definição de cada ínstar foi feita de acordo com as descrições de SKIDMORE (1985) tendo como parâmetros a forma do esqueleto céfalo-faríngeo, a presença de espiráculos anteriores e o número de fendas nos espiráculos anais. $\mathrm{O}$ período de larva de terceiro ínstar na fase de pós-alimentação foi considerado a partir do momento que a larva abandou o frasco de criação, após cessar a alimentação por completo, e terminou ao iniciar a formação do pupário branco. O período de desenvolvimento dos estágios de pré-pupa e pupa iniciou com a retração dos três primeiros segmentos das larvas para dentro do corpo, seguido pela diminuição gradual do corpo e formação do pupário branco e terminando com a emergência dos adultos. Foram utilizadas 270 larvas de terceiro ínstar para a estimativa do período de pós-alimentação e 250 pupas para estimar o período de desenvolvimento e viabilidade dos estágios de prépupa e pupa. A fase de pós-alimentação e o estágio de prépupa + pupa foram observados de 12 em 12 horas.

Os dados referentes ao ciclo de desenvolvimento foram obtidos a partir da média, desvio padrão e variação dos resultados encontrados para cada estágio do ciclo em relação ao período e a frequiência de ocorrência. A viabilidade total e parcial foi obtida conforme PARRA (1996), onde a viabilidade total é um produto da viabilidade de cada estágio ou ínstar.

Larvas e pupas foram depositadas na Coleção de Insetos de Importância Médica e Veterinária, Departamento de Microbiologia e Parasitologia, Universidade Federal de Pelotas (UFPel), Pelotas, RS.

\section{RESULTADOS EDISCUSSÃO}

A primeira eclosão ocorreu 15 horas após a oviposição, sendo que, entre 16 e 20 horas, $61 \%$ das larvas eclodiram (Fig. 1A) com média de 20 horas e coeficiente de variação de $16 \%$ (Tabela I). Este período médio é relativamente maior do que o encontrado por JoHNSON \& VENARD (1957) e menor do que o estimado por RIBEIRo et al. (2000a) para O. aenescens, 16 e 24 horas respectivamente. Synthesiomyia nudiseta (Wulp, 1896) (Muscidae), aos $28^{\circ} \mathrm{C}$, leva, em média, 24 horas para completar o desenvolvimento embrionário, com os adultos sendo criados em dieta à base de açúcar e pedaços de carne bovina (RABINOVICH 1970). A variação encontrada no período de incubação pode 
Tabela I. Desenvolvimento de Ophyra albuquerquei em condições de laboratório à temperatura de $26^{\circ} \mathrm{C} \pm 1{ }^{\circ} \mathrm{C}$ e umidade relativa do ar de $75 \% \pm$ $10 \%$. Número de ovos, larvas e pupários utilizados $\left(\mathrm{N}^{*}\right)$; ** Média ponderada (X); Desvio Padrão (s); Variação (V); Viabilidade (v); Coeficiente de variação $(\mathrm{CV})$; Somatório dos períodos de desenvolvimento médio das larvas $(\Sigma \mathrm{X})$. (a) período da fase de alimentação; (b) período da fase de pósalimentação.

\begin{tabular}{|c|c|c|c|c|c|c|}
\hline \multirow{2}{*}{\multicolumn{2}{|c|}{ Estágio }} & \multicolumn{5}{|c|}{ Período de desenvolvimento (horas) } \\
\hline & & $\mathrm{N}^{*}$ & $\mathrm{X} \pm \mathrm{s} * *$ & $\mathrm{~V}$ & $\mathrm{~V}(\%)$ & $\mathrm{CV}(\%)$ \\
\hline \multicolumn{2}{|c|}{ Ovo } & 275 & $19,93 \pm 3,24$ & $15-27$ & 82,54 & 16,26 \\
\hline & $1^{\circ}$ ínstar & 200 & $29,59 \pm 1,76$ & $24-36$ & 94,50 & 5,95 \\
\hline \multirow[t]{3}{*}{ Larva } & $2^{o}$ ínstar & 200 & $24,25 \pm 4,71$ & $12-36$ & 97,50 & 19,42 \\
\hline & $3^{\circ}$ ínstar ${ }^{\mathrm{a}}$ & 200 & $114,32 \pm 30,36$ & $48-192$ & 94,00 & 26,56 \\
\hline & $3^{o}$ ínstar $^{\mathrm{b}}$ & 270 & $110,73 \pm 35,57$ & $36-204$ & 94,44 & 32,12 \\
\hline \multicolumn{2}{|c|}{ Pupariação } & 250 & $274,42 \pm 38,09$ & $192-372$ & 94,40 & 13,88 \\
\hline \multirow{2}{*}{\multicolumn{2}{|c|}{$\begin{array}{l}\text { EX Larva } \\
\text { Ovo-adulto }\end{array}$}} & --- & 278,89 & $120-468$ & 81,79 & --- \\
\hline & & - & 573,24 & $325-867$ & 63,73 & - \\
\hline
\end{tabular}

ser causada pelo tempo de retenção dos ovos no útero da fêmea (Howe 1966), pela variação inter-específica ou mesmo entre populações, estando relacionada à dieta utilizada para os adultos (PARRA 1991). Pode também ser causada pelos intervalos de observações ou mesmo, devido à plasticidade fenotípica inerente a cada espécie, influenciando a estimativa das médias destes períodos.

Quanto à dieta para adultos, diferenças na proporcionalidade de proteínas e carboidratos têm significativa influência na viabilidade dos ovos (Howe 1966). Muscina stabulans Fallén, 1825 (Muscidae) (P.B. Ribeiro, comunicação pessoal), O. aenescens (Ribeiro et al. 2000a) e S. nudiseta (KRÜGER et al. 2002), cujos adultos foram alimentados com a mesma dieta oferecida aos adultos de $O$. albuquerquei, apresentaram viabilidade de $93 \%, 89 \%$ e $79 \%$, respectivamente. Entre as espécies mencionadas acima, a viabilidade foi próxima à encontrada para $O$. albuquerquei (Tabela I), confirmando que a dieta oferecida aos adultos dessas espécies pode ser ofertada a outras espécies de Muscidae que possuam hábitos semelhantes.

A larva de primeiro ínstar, ao eclodir, possui o hábito de se dispersar rapidamente pelo substrato, o que pode dificultar a sua observação, fato constatado para Sarconesia chlorogaster (Wiedemann, 1830) (Calliphoridae), sendo este comportamento uma possível estratégia de proteção de predadores e de agressões (BONATTO 1996). A exploração realizada por larvas de primeiro ínstar seria utilizada como um mecanismo que minimizasse não somente a predação, mas também o canibalismo, principalmente entre aquelas espécies que sejam predadoras, como no caso de O. albuquerquei. Outro fator que pode levar à exploração do substrato deve-se à escassez de alimento que leva à atividade de forrageamento (Holuing 1961) o que, neste último caso, deve ser desconsiderado, já que este ínstar apresentou alta viabilidade com o menor coeficiente de variação entre todos os ínstares.

O período médio de desenvolvimento foi de 29 horas (Tabela I), sendo que entre 24 e 30 horas, $91 \%$ das larvas passaram ao segundo ínstar (Fig. 1B). Ao passar para o segundo ínstar, as larvas formaram pequenos agrupamentos com incessante processo alimentar. Este comportamento é essencial para a transformação do meio de cultura e conseqüentemente para o pleno desenvolvimento larval. Isto foi observado em Chrysomya albiceps Wiedemann, 1818 (Calliphoridae) (QuEIROZ $\&$ Milward-De-Azevedo 1991) e S. chlorogaster (Bonatto 1996). Após 12 horas, as primeiras larvas começaram a passar para o terceiro ínstar e 24 horas após, 97,5\% das 200 larvas completaram seu desenvolvimento (Tabela I), sendo o intervalo de 12 a 24 horas o que obteve maior freqüência, com $84,6 \%$ (Fig. 1C). O período médio de desenvolvimento foi de 24 horas (Tabela I). Este período foi a metade do observado para $S$. nudiseta nas mesmas condições (KRÜGER et al. 2002) apesar de O. albuquerquei apresentar viabilidade muito superior.

O terceiro ínstar foi o que apresentou maior período de desenvolvimento, sendo que a fase alimentar deste ínstar tem, pelo menos, duas classes modais: entre 60 e 84 horas e entre 132 e 156 horas após a passagem do segundo para o terceiro ínstar (Fig. 1D). Possivelmente por isso, o coeficiente de variação apresentou-se tão alto em relação aos outros ínstares. O período médio foi de 114 horas, com variação de 48 a 192 horas (Tabela I). O período médio de desenvolvimento desta fase para S. nudiseta é um pouco mais longo que o dobro do tempo necessário para que $O$. albuquerquei complete esta fase, em torno de 233 horas e com viabilidade aproximada (KRÜGER et al.2002).

Na fase pós-alimentar do terceiro ínstar, a classe com maior frequiência de formação dos pupários, ocorreu entre 96 e 120 horas com mais de 70 indivíduos (Fig. 1E). O período médio de desenvolvimento desta fase foi próximo da fase anterior, 111 horas, sendo a variação maior. Diversos autores (D'AlMEIDA et al. 1999; RibeIRo et al. 2000a, entre outros) consideram esta fase como o estágio de pré-pupa, provavelmente por apresentar um comportamento diferenciado dos outros ínstares. A fase pós-alimentar do terceiro ínstar é caracterizada pelo abandono do meio de criação pela larva já madura. Nesta fase, ela percorre o substrato a fim de encontrar um local apropriado para iniciar o processo de pupariação, ou seja, a formação do pupário. $\mathrm{Na}$ 


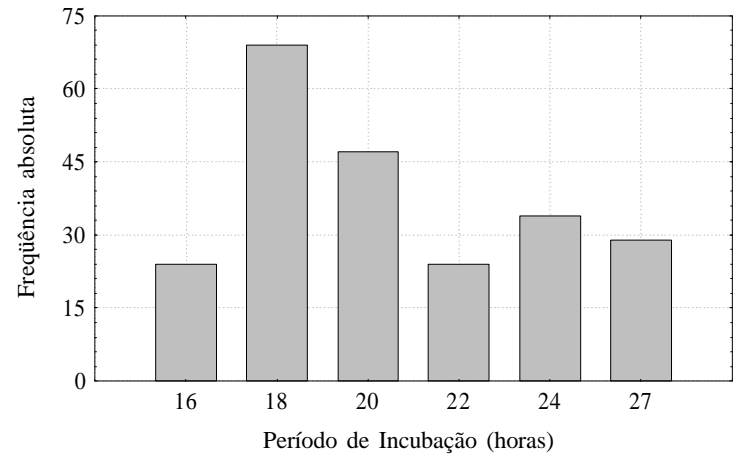

A

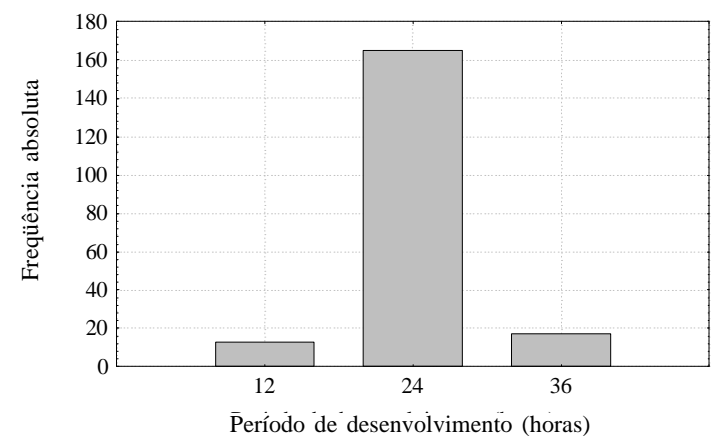

$\mathrm{C}$

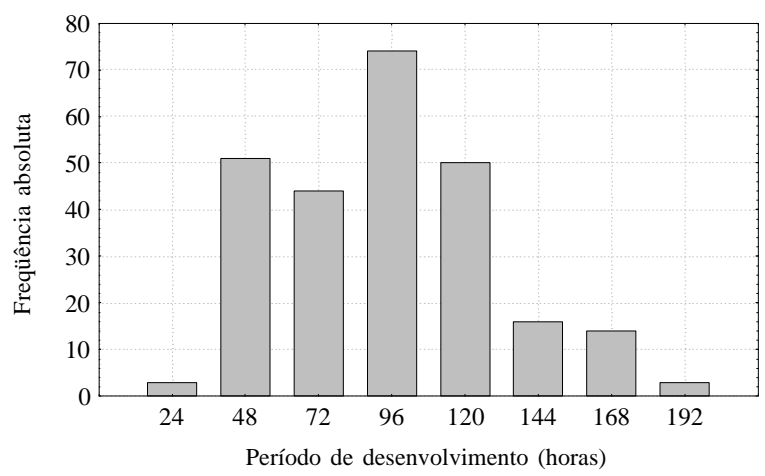

$\mathrm{E}$

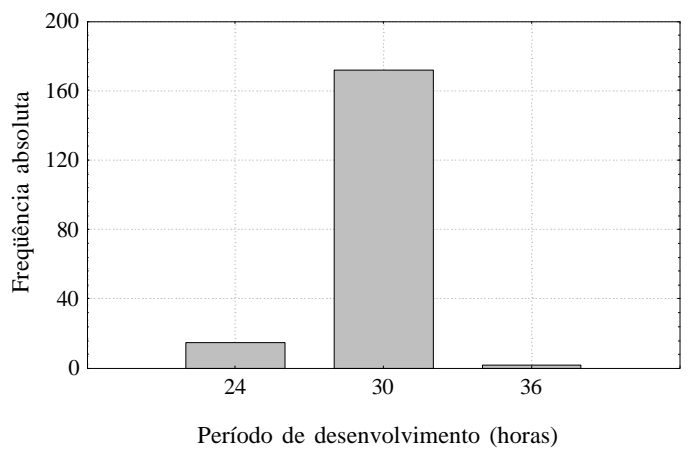

B

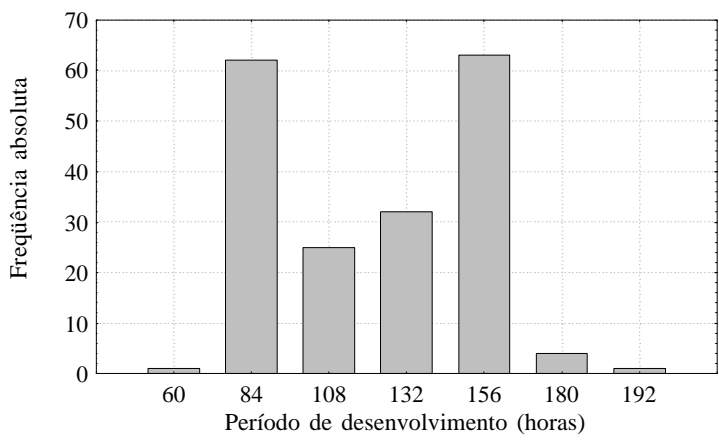

$\mathrm{D}$

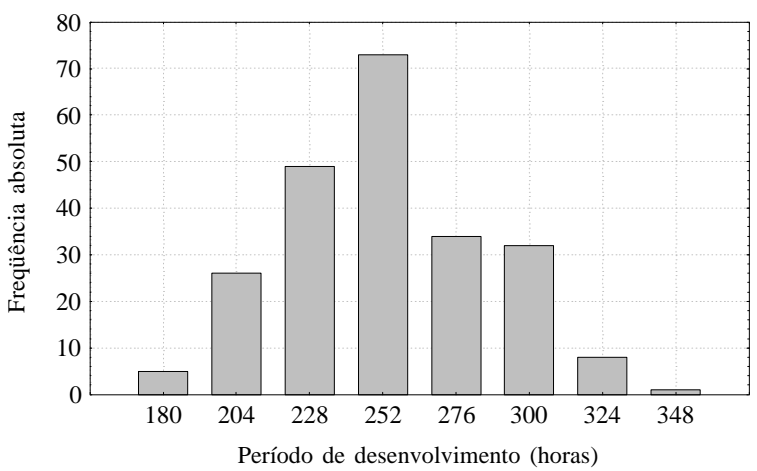

$\mathrm{F}$

Fig. 1. Distribuição de freqüência do período de desenvolvimento de Ophyra albuquerquei a $26^{\circ} \mathrm{C}$, UR de $75 \%$ e fotoperíodo de 12 horas. A, estágio de ovo; B, larva de primeiro ínstar; C, larva de segundo ínstar; D, larva de terceiro ínstar na fase alimentar; E, larva de terceiro ínstar na fase pósalimentar; F, estágio de pré-pupa + pupa.

fase pós-alimentar, a larva pára de se alimentar irreversivelmente, passando então a esvaziar o intestino, finalizando com a formação do pupário branco. Nesta fase, não há evidência de transformação morfológica para que ela seja considerada como um estágio à parte (FRAENKEL \& BHASKARAN 1973).

O estágio de larva de O. albuquerquei teve duração de 279 horas (11-12 dias), com variação mínima de 120 horas (5 dias) e máxima de 468 horas (19,5 dias). Este período é muito superior ao encontrado para $O$. aenescens que pode ser de quatro
(Johnson \& Venard 1957), cinco (D'Almeida et al. 1999) ou de sete dias (Ribeiro et al. 2000a). Mesmo entre outras espécies de Muscidae, O. albuquerquei possui período de desenvolvimento das larvas muito superior ao de M. stabulans, seis dias (P. B. Ribeiro, comunicação pessoal) e ao de $S$. nudiseta, 8,5 dias (RABINOvich 1970), apesar desta espécie apresentar neste estágio um desenvolvimento de 307 horas (12-13 dias) em dieta artificial (KRÜGER et al. 2002).

Todos os ínstares apresentaram alta viabilidade, culminando 
em uma viabilidade de $82 \%$ para o estágio de larva, sendo maior que a encontrada para O. aenescens (cerca de 78\%). Entre os estágios, é no de larva que geralmente ocorre a menor taxa de sobrevivência (Tabela I), atribuindo-se isto à maior dependência deste estágio em relação ao ambiente, sendo mais sensível às modificações bióticas e abióticas (RIBEIRo et al. 2000a).

O estágio de pupa apresentou período de desenvolvimento próximo ao de larva (cerca de 274 horas) com variação de 192 (oito dias) a 372 horas (15,5 dias) (Tabela I). Em torno de $31 \%$ das emergências ocorrem na classe de 252 e 276 horas após o inicio deste estágio, sendo que até 300 horas, mais de $79 \%$ das emergências já havia ocorrido (Fig. 1F). Como ocorre no estágio de larva, o estágio de pupa de $O$. albuquerquei é o que apresenta maior período de desenvolvimento quando comparado à $O$. aenescens, cinco (JoHNSON \& VENARD 1957) e 10 dias (D'Almeida et al. 1999; RiBeIRo et al. 2000a), M. stabulans, nove dias (P. B. Ribeiro, comunicação pessoal) e $S$. nudiseta, oito dias (RABINOVICH 1970). É neste estágio que ocorre a maior viabilidade entre todas as fases de desenvolvimento.

Considerando-se a somatória das médias dos períodos de desenvolvimento de todos os estágios de O. albuquerquei, o ciclo de ovo a adulto desta espécie foi de 573 horas (23-24 dias) com variação de 13,5 a 36 dias e viabilidade total de $63,73 \%$ (Tabela I). Em relação a este ciclo, nesta temperatura, o período varia de 16 dias para M. stabulans com viabilidade de $89 \%$ (P. B. Ribeiro, comunicação pessoal) a 18 dias para O. aenescens (Ribeiro et al. 2000a) e S. nudiseta (RABINOvich 1970) com viabilidade próxima à encontrada para esta espécie. Em dieta artificial, $S$. nudiseta pode chegar a 27 dias de duração para este ciclo, mas com baixa viabilidade, cerca de $49 \%$ (KRÜGER et al.2002).

Muitas das diferenças entre as espécies quanto ao período e viabilidade dos seus estágios estão fortemente relacionadas à dieta utilizada para sua manutenção. Espécies com larvas que possuem hábitos carnívoros, como o caso das larvas de terceiro ínstar de Ophyra spp., S. nudiseta e M. stabulans (SKIDMORE 1985), necessitam de uma alta proporção de proteínas, caso contrário pode haver um prolongamento dos períodos de desenvolvimento, bem como uma forte influência na viabilidade. Uma larva tende a escolher um alimento apropriado para consumi-lo em condições balanceadas a fim de promover um ótimo crescimento e desenvolvimento, dando origem a um adulto que seja reprodutivamente competitivo (PARRA 1991). A duração e viabilidade larval; a duração, viabilidade e peso das pupas; a duração e viabilidade do período pós-embrionário; o ritmo das emergências e razão sexual; e, finalmente, o tamanho dos adultos, podem ser utilizados como parâmetros de medida de uma dieta artificial (D’Almeida \& Mello 1995). Baseado principalmente na viabilidade e nos períodos de desenvolvimento de Ophyra albuquerquei, constata-se que a dieta utilizada para criação das larvas desta espécie possui ótimos atributos qualitativos e quantitativos, viabilizando a manutenção da criação desta espécie em laboratório.

Entre as espécies que tiveram os períodos de desenvolvimento comparados com O. albuquerquei, $S$. nudiseta foi a que apresentou maior semelhança quanto à duração dos estágios, provavelmente por apresentarem exigências nutricionais semelhantes. Adultos de $O$. albuquerquei visitam carcaça de Rattus novergicus Linnaeus, 1758 (Rodentia, Muridae), oito dias após a primeira exposição (Moura et al. 1997, 1998) como ocorre com S. nudiseta (BOHART \& Gressit 1951). De acordo com SKIDMORE (1985), esta espécie coloniza os substratos após a chegada de $M$. stabulans e $M$. domestica, quando o teor de proteínas bruta já está reduzido.

A dieta oferecida às larvas de $O$. albuquerquei possui características ótimas que viabilizam a manutenção desta espécie em laboratório para realização de trabalhos futuros sobre capacidade de predação, competição e exigências térmicas.

Agradecimentos. Ao Conselho Nacional de Desenvolvimento Científico e Tecnológico $(\mathrm{CNPq})$ pelas bolsas concedidas a RFK (processo 130.415/00-6) e a CJBC (processo 304.148/2002-4). Ao MédicoVeterinário Paulo Renato Peixoto Costa (in memorian) pelo apoio na manutenção das colônias em laboratório. À Profa. Dra. Marines Garcia (Departamento de Botânica, Universidade Federal de Pelotas) pelas sugestões para o abstract. Aos consultores anônimos da RBE pelas sugestões e críticas.

\section{REFERÊNCIAS}

Anderson, J. R. \& J. H. Poorbaugh. 1964. Biological control possibility for house flies. California Agriculture 18(9): 2-4.

Avancini, R. M. P. \& G. A. R. Silveira. 2000. Age structure and abundance in populations of muscoid flies from poultry facility in Southeast Brazil. Memórias do Instituto Oswaldo Cruz 95(2): 259-264.

Axtell, R. C. 1999. Poultry integrated pest management: status and future. Integrated Pest Management Reviews 4: 53-73.

Betke, P.; T. Hiepe; P. Muller; H. Schultka \& H. Schumann. 1989. Biological control of Musca domestica with Ophyra aenescens on pig production enterprises. Monastshefte for Veterinarmedizin 44: 842-844.

Bohart, G. E. \& J. L. Gressit. 1951. Filth-inhabiting flies of Guam. Honolulu, Bernice P. Bishop Museum, Bulletin 204, vii + 152 p.

Bonatto, S. R. 1996. Ciclo de vida de Sarconesia chlorogaster (Wiedemann) (Diptera, Calliphoridae, Toxotarsinae), criada sob condições de laboratório em dieta artificial. Revista Brasileira de Zoologia 13(3): 685-706

Carvalho, C. J. B. De; A. C. Pont; M. S. Couri; D. Pamplona \& S. M. Lopes. 1993. Part II. Muscidae. In: C. J. B. De Carvalho (Ed.). A catalogue of the Fanniidae and Muscidae (Diptera) of the Neotropical Region. São Paulo, Sociedade Brasileira de Entomologia, 201 p.

Carvalho, L. M. L.; P. J. Thyssen; A. X. Linhares \& F. A. B. Palhares. 2000. A checklist of arthropods associated with pig carrion and human corpses in southeastern Brazil. Memórias do Instituto Oswaldo Cruz 95(1): 135-138.

Costa, P. R. P.; R. L. Franz; E. E. S. Vianna \& P. B. Ribeiro. 2000. Synanthropy of Ophyra spp (Diptera, Muscidae) in Pelotas, RS, Brazil. Revista Brasileira de Parasitologia Veterinária 9(2): $165-168$.

D’Almeida, J. M. 1988. Substratos utilizados para a criação de dípteros caliptratos em uma área urbana do município do Rio de Janeiro. Memórias do Instituto Oswaldo Cruz 83(2): 201-206.

D’Almeida, J. M. 1989. Substratos utilizados para a criação de dípteros caliptratos no jardim zoológico do Rio de Janeiro (RIO-ZOO). Memórias do Instituto Oswaldo Cruz 84(2): 257-264.

D’Almeida, J. M.; C. Borges \& C. A. Gonçalves. 1999. Desenvolvimento pós-embrionário de Ophyra aenescens (Wiedemann, 1830) (Diptera: Muscidae) em diferentes dietas, sob condições de laboratório. Memórias do Instituto Oswaldo Cruz 94(1): 123-126. 
D’Almeida, J. M. \& R. P. Mello. 1995. Eficiência de variadas dietas na criação de Chrysomya megacephala (Fabricius, 1774) e Chrysomya putoria (Wiedemann, 1818) (Diptera: Calliphoridae), sob condições de laboratório. Entomología y Vectores 2: 95-106.

Fraenkel, G. \& G. Bhaskaran. 1973. Pupariation and pupation in Cyclorrhaphous flies (Diptera): Terminology and interpretation. Annals of the Entomological Society of America 66(2): 418422.

Geden, C. J.; R. E. Stinner \& R. C. Axtell. 1988. Predation by predators of the house fly in poultry manure: effects of predator density, feeding history, interspecific interference, and field conditions. Environmental Entomology 17(2): 320-329.

Holling, C. S. 1961. Priciple of insect predation. Annual Review of Entomology 6: 163-182.

Hogsette, J. A. \& F. Washington. 1995 Quantitative mass production of Hydrotaea aenescens (Diptera: Muscidae). Journal of Economic Entomology 88(5): 1238-1242.

Howe, R. W. 1966. Temperature effects on embryonic development in insects. Annual Review of Entomology 11: 15-42.

Johnson, W. T. \& C. E. VenARD. 1957. Observations on the biology and morphology of Ophyra aenescens (Diptera: Muscidae). The Ohio Journal of Science 57(1): 21-26.

Krüger, R. F; P. B. Ribeiro; C. J. B. De Carvalho \& P. R. P. Costa. 2002. Desenvolvimento de Synthesiomyia nudiseta (Diptera, Muscidae) em laboratório. Iheringia, Série Zoologia, 92(4): 25-30.

Moura, M. O.; C. J. B. de Carvalho \& E. L. A. Monteiro-Filho. 1997. A preliminary analysis of insects of medico-legal importance in Curitiba, State of Paraná. Memórias do Instituto Oswaldo Cruz 92(2): 269-274.

Moura, M. O.; C. J. B. de Carvalho \& E. L. A. Monteiro-Filho. 1998. Carrion attendant arthropods in southern Brazil. Ciência e Cultura 50(5): 377-381.

Olckers, T. \& P. E. Hulley. 1984. Facultative predation of house fly by larvae of Ophyra capensis (Wiedemann) (Diptera: Muscidae). Journal of Entomology Society of South African 47(2): 231237

Pamplona, D. \& M. S. Couri. 1989. Revisão das espécies neotropicais de Ophyra Robineau-Desvoidy, 1830 (Diptera, Muscidae, Azeliinae). Memórias do Instituto Oswaldo Cruz 84(supl. IV): 419-429.

Parra, J. R. P. 1991. Consumo e utilização de alimentos por insetos, p. 9-65. In: A. R. PANIZZI \& J. R. P. PARRA. (Eds.). Ecologia nutricional de insetos e suas implicações no manejo de pragas. São Paulo,
Editora Manole, xii+359 p.

PARra, J. R. P. 1996. Técnicas de criação de insetos para programas de controle biológico. Piracicaba, Escola Superior de Agricultura Luiz de Queiroz, 137 p.

PeCK, J. H. 1969. Arthropod predators of immature Diptera developing in poultry droppings in northern California. Journal of Medical Entomology 6(2): 168-171.

Queiroz, M. M. DE C. \& E. M. V. Milward-DE-Azevedo. 1991. Técnicas de criação e alguns aspectos da biologia de Chrysomya albiceps (Wiedemann) (Diptera, Calliphoridae), em condições de laboratório. Revista Brasileira de Zoologia 8(1/2/3/4): 75-84.

Rabinovich, J. 1970. Vital statistics of Synthesiomyia nudiseta (Diptera: Muscidae). Annals of the Entomological Society of America 63(3): 749-752.

Ribeiro, P. B.; C. J. B. de Carvalho; P. R. P. Costa \& P. Silveira-Júnior. 2000a. Desenvolvimento de Ophyra aenescens Wiedemann, 1830 (Diptera, Muscidae, Azeliinae), em diferentes temperaturas, em condições de laboratório. Revista Brasileira de Agrociência 6(1): 80-87.

Ribeiro, P. B.; C. J. B. De Carvalho; L. Pinto \& P. Silveira-Júnior. 2000 b. Flutuação populacional das espécies de Ophyra Robineau-Desvoidy (Diptera, Muscidae, Azeliinae) na região de Pelotas, RS. Arquivos do Instituto Biológico 67(2): 205-214.

SchumanN, H. 1989. Biologische bekampfung an beispiel des stubenfliegen antagonistan Ophyra aenescens. Wissenschaftliche Zeitschrift der Humboldt Universitat zu Berlin, Mathematisch Naturwissen Chaftliche Reihe 38(4): 415-419.

Silveira-neto, S.; O. NaKano; D. Barbin \& N. A. Villa Nova. 1976. Manual de Ecologia dos Insetos. São Paulo, Ed. Agronômica Ceres, 419 p.

Skidmore, P. 1985. The biology of the Muscidae of the world. Dordrecht, Dordrecht Kunk Publishers, 550 p.

Tantawi, T. I.; E. M. El-Kady; B. Greenberg \& H. A. El-chaffar. 1996. Arthropod sucession on exposed rabbit carrion in Alexandria, Egypt. Journal of Medical Entomology 33(4): 566-580.

Tsankova, R. N. \& V. I. LuvchiEv. 1993. Laboratory investigations on the larval zoophagy of Ophyra capensis - an antagonist of $\mathrm{Musca}$ domestica. Applied Parasitology 34(3): 221-228.

Youngman, R. R.; E. C. TuRNER-JR \& P. L. ReuZler. 1991. Instructions on insectary establishment, mass rearing and release of ophyra aenescens. A house fly predator. Virginia, Virginia Cooperative Extension, 4 p. 